\title{
Complementary sestamibi scintigraphy and ultrasound for primary hyperparathyroidism
}

\author{
Yang $Z^{1,3 *}$ Li AY', Alexander $G^{3}$ and Chadha $M^{3}$ \\ ${ }^{1}$ Department of Radiology, Louisiana State University Health Sciences Center/University Health, Shreveport, LA, USA \\ ${ }^{2}$ School of Medicine, Louisiana State University Health Sciences Center Shreveport, 1501 Kings Highway, Shreveport, LA, USA \\ ${ }^{3}$ Department of Radiology, Louisiana State University Health Sciences Center/University Health, LA, USA
}

\begin{abstract}
Background: With the increasing adoption of minimally invasive parathyroidectomy techniques, the dual use of sestamibi scintigaphy (MIBI) and Ultrasound (US) has become more important in the preoperative evaluation of primary hyperthyroidism. The results presented here arose from a Practice Quality Improvement project (PQI) at our institution, which demonstrated that when compared against surgical outcomes, the use of both MIBI and US enhanced the detection of enlarged parathyroid glands. This project corroborates the complementary nature of these two imaging modalities in the preoperative localization of enlarged parathyroid glands.

Methods: Fifty-six consecutive cases that coupled the usage of MIBI and US were included during the duration of our PQI project from January to November of 2014. Patients in every case were diagnosed with typical Primary Hyperparathyroidism (PHPT) accompanied by the elevation of Parathyroid Hormone (PTH) and calcium levels, with the exception of one case.

Results: In 34 out of 56 cases, the parallel use of preoperative MIBI and US resulted in consistent findings between the two modalities. The remaining 22 cases demonstrated that the limitations of one modality can be overcome by the advantages of the alternate modality.

Conclusion: MIBI provides guidance for the interpretation of US data, especially in the context of ectopic parathyroid glands, small parathyroid adenomas, and concurrent thyroid nodules. US offers detailed anatomic information and supports the diagnostic confidence of interpreting MIBI scans. US can be especially helpful in patients with more than one enlarged parathyroid gland. In the majority of cases, the dual utilization of MIBI and US was able to successfully overcome the inherent limitations of each modality when employed alone
\end{abstract}

\section{Introduction}

With the development of minimally invasive parathyroidectomy (MIP) procedures, sestamibi scintigraphy (MIBI) and ultrasound (US) have emerged as the predominant techniques in the preoperative evaluation of primary hyperparathyroidism (PHP). Not only are MIBI and US the most informative modalities for the detection of abnormal parathyroid glands, but they are potentially complementary [1-3]. The precise localization of enlarged parathyroid glands before embarking on major neck surgery has become indispensable to the management of PHPT. This article presents the results from a Practice Quality Improvement project (PQI) at our institution, which demonstrated that when compared against surgical outcomes, the use of both MIBI and US enhanced the detection of enlarged parathyroid glands. This project improved our diagnostic confidence and accuracy and supported the proposed complementary nature of these two imaging modalities in the visualization of the etiology of PHPT.

\section{Methods}

Fifty-six consecutive cases that coupled the usage of MIBI and US were included for the duration of our PQI project from January to November of 2014. Patients in every case were diagnosed with typical PHPT accompanied by the elevation of PTH and calcium levels, with the exception of one case (Figure 1). To improve the correlation between MIBI and US in the preoperative localization of parathyroid adenomas, we held a consultation session between the nuclear medicine physicians, US radiologists, and US technologists on each case prior to patient discharge. A repeat US scan was performed if the results from both scans were inconsistent with each other.

\section{Results}

In 34 of the 56 cases, the parallel use of preoperative MIBI and US resulted in consistent findings between the two imaging modalities. A typical feature of parathyroid adenomas on MIBI is a focal area of persistently increased uptake in the region of the thyroid gland. On US, the characteristic appearance of parathyroid adenomas is a homogeneously hypoechoic focal area with a feeding vessel and a peripheral distribution of vascularity (Figure 2A). The remaining 22 cases demonstrate that the limitations of one modality can be overcome

Correspondence to: Zhiyun Yang, Department of Radiology, Louisiana State University Health Sciences Center/University Health, Shreveport, LA, USA; Tel: 318-675-6214; Fax: 318-675-6244; E-mail: zyang@lsuhsc.edu

Key words: sestamibi scintigraphy (MIBI), ultrasound (US), primary hyperparathyroidism (PHPT), parathyroid hormone (PTH), enlarged parathyroid glands, parathyroid adenoma, minimally invasive parathyroidectomy

Received: December 13, 2016; Accepted: January 10, 2017; Published: January 13,2017 

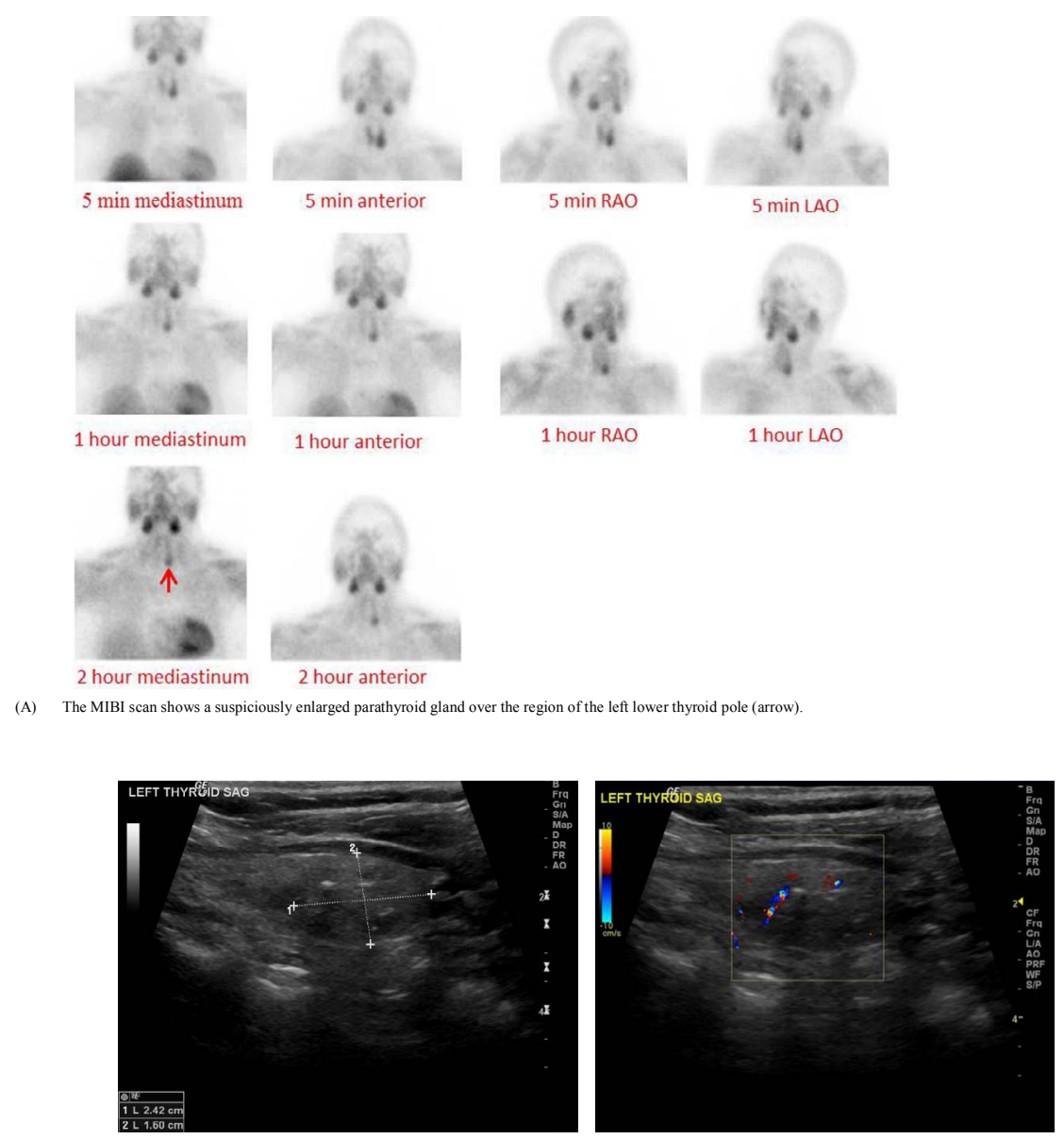

(B) The US scan shows a hyperechoic nodule with calcifications and minimal vascularity within the left inferior thyroid pole, which is indicative of a thyroid nodule rather than a parathyroid gland. Subsequent surgery revealed a thyroid nodule but did not show any enlarged parathyroid gland in this area.

Figure 1. A 71-year-old female presented with a PTH level of $69.5 \mathrm{pg} / \mathrm{ml}$ and a calcium level of $8.8 \mathrm{mg} / \mathrm{dl}$, which is clinically suspicious for PHPT.

by the advantages of the alternate modality.

The limitations of MIBI include: 1) false-positive findings due to thyroid nodules (Figure 1); 2) false-negative or low confidence results due to the relatively small size of enlarged parathyroid gland(s) (Figure 3); 3) suboptimal results due to body habitus, motion, patient intolerance to lengthy procedures, or an adverse tracer reaction (Figure 2B); 4) information arising from a predominant, hyperfunctioning parathyroid gland that overwhelms the uptake activity from the other non-dominant, enlarged parathyroid glands (Figure 4); 5) the inability to portray an accurate relationship between an enlarged parathyroid gland and its surrounding structures (Figure 5); and 6) the inability to detail concurrent thyroid nodules (Figure 6).

The limitations of US scans include: 1) operator-dependent analyses and 2) the inability to detect ectopic parathyroid glands (Figure 7).

\section{Discussion}

PHTP is a common disease that is characterized by a destructive and progressive process involving multiple organ systems, for which the only cure is surgery [3]. The traditional surgical therapy, bilateral four-gland exploration, has been gradually replaced by unilateral and more focused surgical approaches such as MIP techniques at most medical centers, including our institution [1]. A successful MIP requires accurate localization of enlarged parathyroid glands by imaging modalities prior to surgery. MIBI scans are the most commonly used imaging technique. The sensitivity of MIBI for detecting parathyroid adenomas ranges widely, from 54 to $96 \%$, mainly as a result of discrepancies in imaging protocols [2]. The significant limitations of MIBI are its low resolution and poor anatomic localization. Conversely, US is an imaging modality with high resolution, which can yield adequate visualization of anatomic details. However, US is limited by its operator-dependent image acquisition and analysis as well as the inability to detect ectopic parathyroid glands. Through our PQI project, we noted our implementation of the following methods to be helpful in overcoming the deficiencies of US: 1) placement of the American Institute of Ultrasound (AIUM) practice guidelines for performing thyroid and parathyroid ultrasound examinations in US scan rooms; 2) incorporation of educational lectures along with this written guide for US technologists, US physicians, and residents; 3) designation of experienced US physicians and technologists to guide the performance of US when warranted; 4) communication with clinicians on the benefits of carrying out (and thus ordering) US and MIBI scans on the same day; 5) acquisition of the MIBI scan following ultrasonography for every patient with PHPT; 6) consultation between the NM physician(s) and US radiologist(s) to reach a consensus on the diagnosis before the patient is discharged; and 7) performance of a repeat US if results from the MIBI and US scans were inconsistent with each other. 

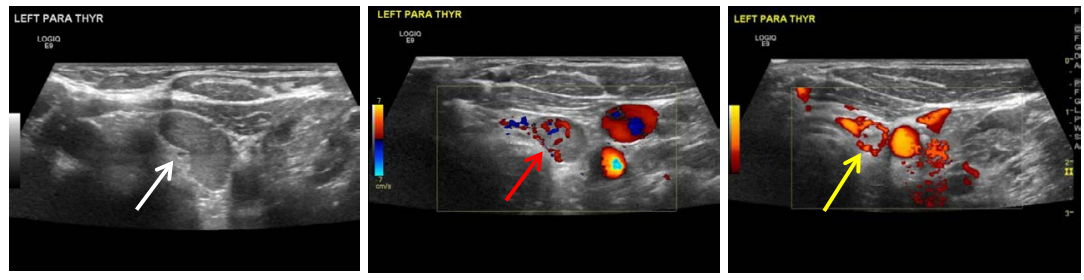

(A): The US scan shows a large hypoechoic focal area (white arrow) with an extrathyroidal feeding vessel (red arrow) and peripheral distribution of vascularity (yellow arrow) in the area of the left inferior thyroid pole. This US scan demonstrates the typical appearance of an enlarged parathyroid gland.

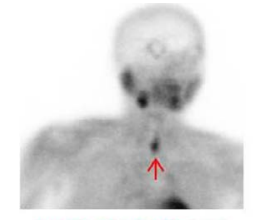

1 hour mediastinum

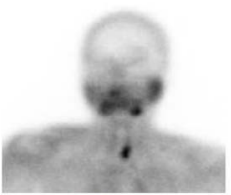

1 hour anterior

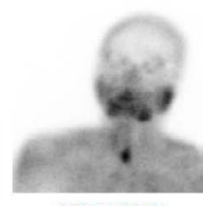

1 hour LAO

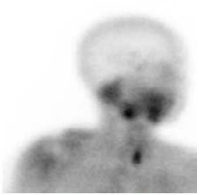

1 hour RAO

(B): The MIBI images show a focal area of increased uptake over the inferior pole of the left thyroid lobe and are suspicioss for an enlarged parathyroid gland. However, additional routine images (the five-minute and the delayed two-hour scans) could not be obtained, because the patient could not tolerate the duration of the scan. Despite the limited MIBI data, findings on both US and MIBI support an enlarged parathyroid gland.

Figure 2. 49-year-old female presented with a PTH level of $1123.5 \mathrm{pg} / \mathrm{ml}$ and a calcium level of $12.8 \mathrm{mg} / \mathrm{dl}$.

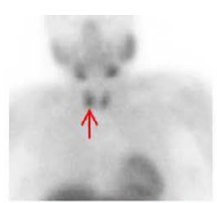

5 min mediastinum

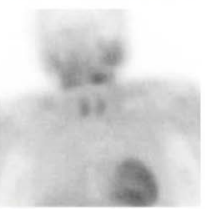

1 hour mediastinum

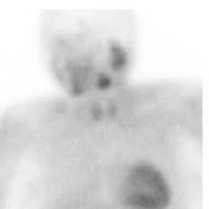

2 hour mediastinum

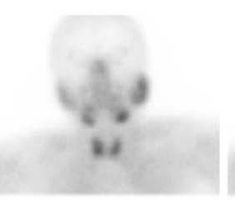

5 min anterior

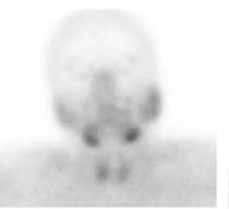

1 hour anterior

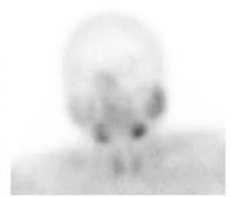

2 hour anterior

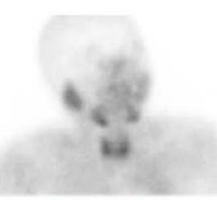

5 min RAO

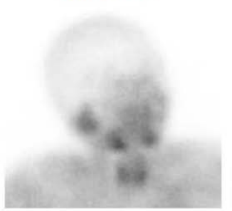

1 hour RAO

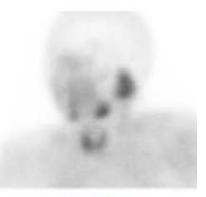

$5 \min$ LAO

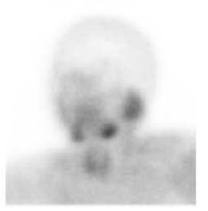

1 hour LAO

(A): The MIBI image is suspicious for an enlarged parathyroid gland over the inferior pole of the right thyroid lobe (arrow). However, this diagnosis is associated a low confidence level due to the relatively small size of this gland.
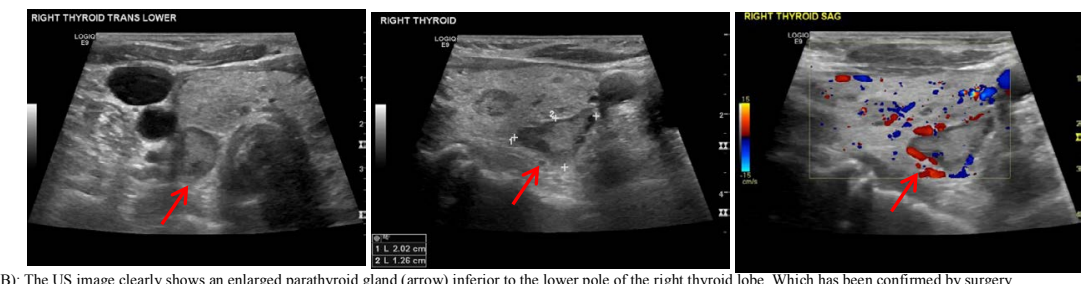

Figure 3. A 49-year-old female presented with a PTH level of $192.5 \mathrm{pg} / \mathrm{ml}$ and a calcium level of $11.1 \mathrm{mg} / \mathrm{dl}$. 


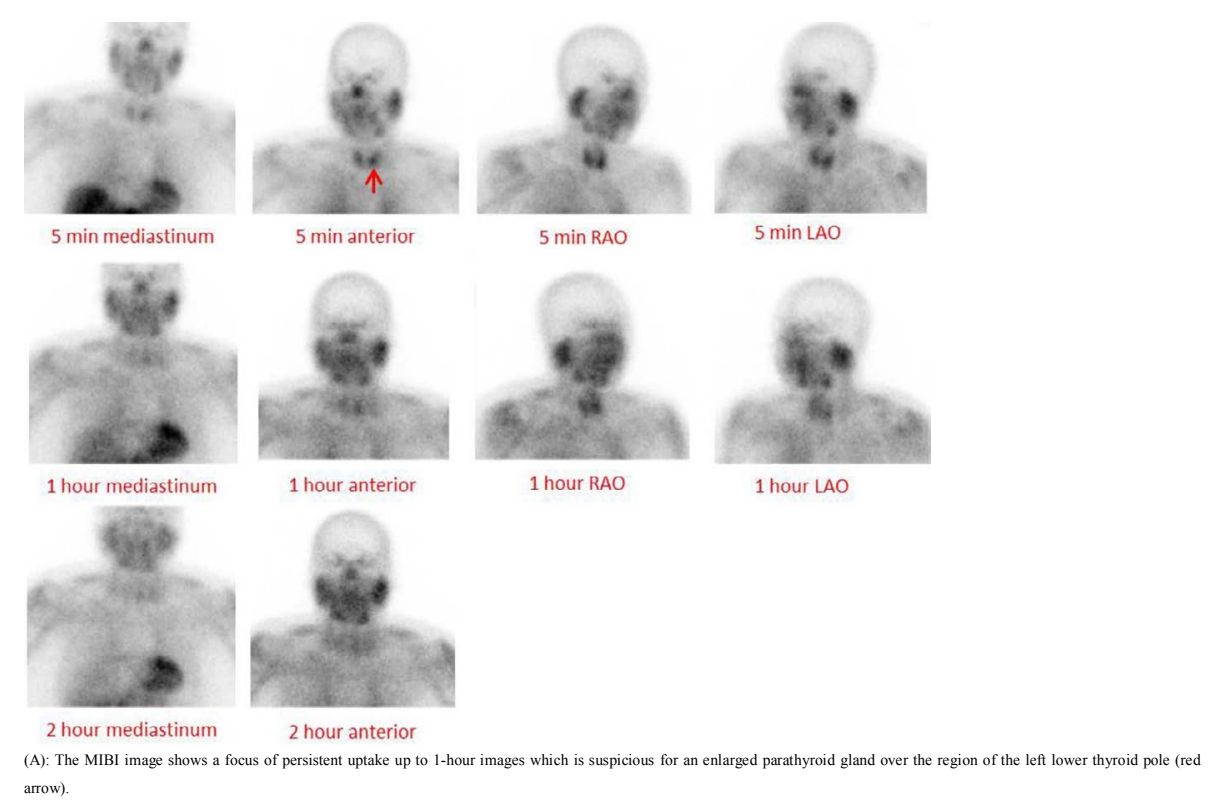
arrow).

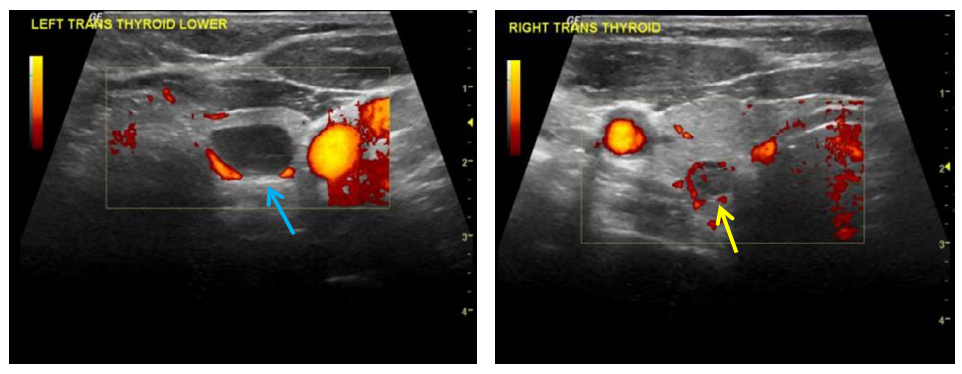

(B): The US images show two enlarged parathyroid glands: an enlarged left inferior parathyroid gland (blue arrow) and a relatively small enlarged right superior parathyroid gland (yellow arrow), which is not visualized on the MIBI scan.

Figure 4. A 72-year-old female presented with a PTH level of $141 \mathrm{pg} / \mathrm{ml}$ and a calcium level of $10.3 \mathrm{mg} / \mathrm{dl}$.
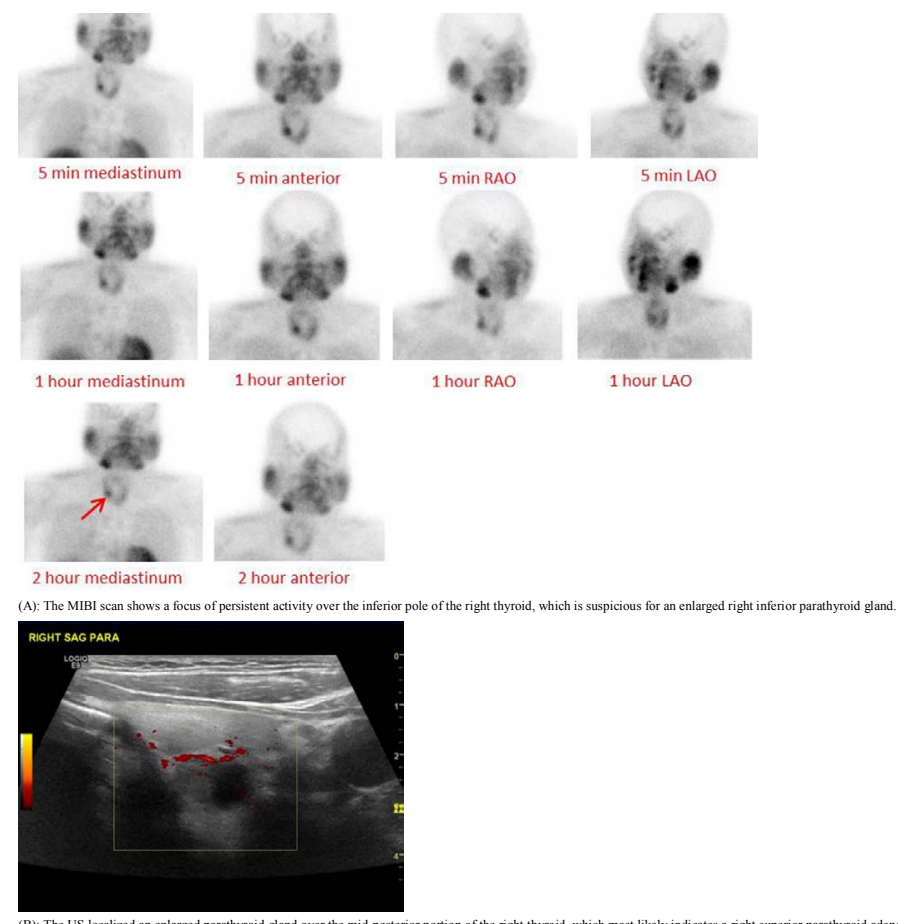

surgical findings supported the US reathyorid gland over the mid-posterior portion of the righ

Figure 5. A 57-year-old female presented with a PTH level of $500 \mathrm{pg} / \mathrm{ml}$ and a calcium level of $10.9 \mathrm{mg} / \mathrm{dl}$. 


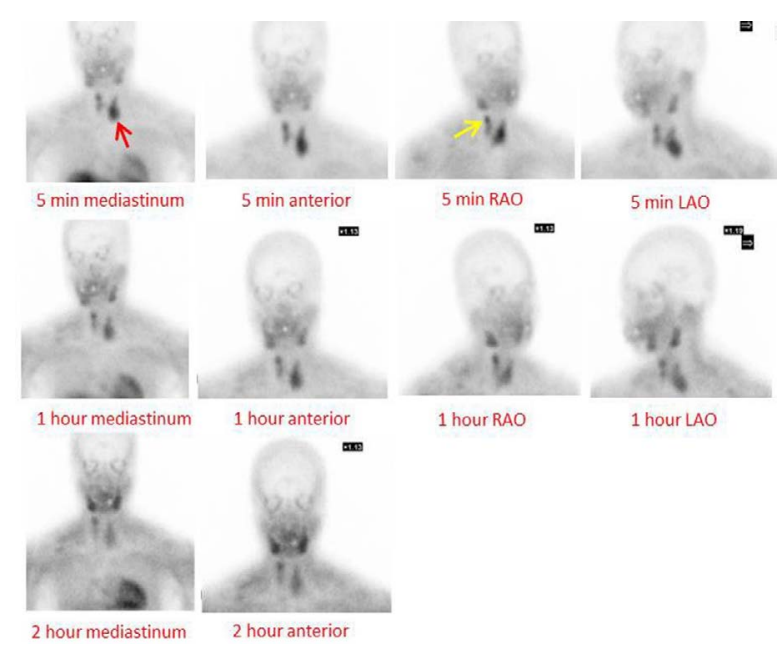

2 hour mediastinum 2 hour anterior

(A): The MIBI images show two foci of persistently increased uptake, with one focus over the right upper thyroid pole and the other focus over the inferior left thyroid. Both may represent enlarged parathyroid glands.
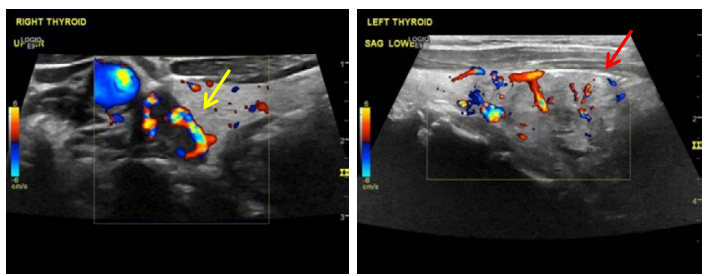

(B): The US scan shows an enlarged parathyroid gland posterior to the rig

Figure 6. A 59-year-old female presented with a PTH level of $344.5 \mathrm{pg} / \mathrm{ml}$ and a calcium level of $11.5 \mathrm{mg} / \mathrm{dl}$.
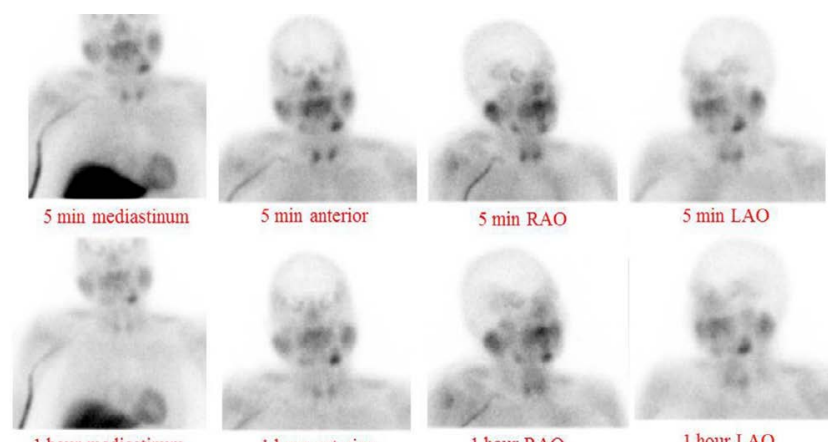

$5 \min \mathrm{RAO}$

$5 \min \mathrm{LAO}$

1 hour mediastinum

1 hour anterior
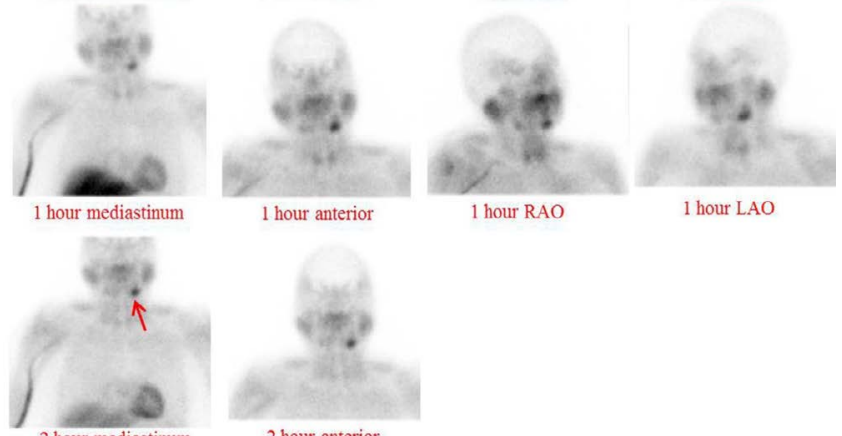

1 hour RAO

1 hour LAO

2 hour mediastinum

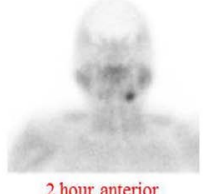

(A): The MIBI images reveal a suspected ectopic parathyroid gland over the left submandibular gland.

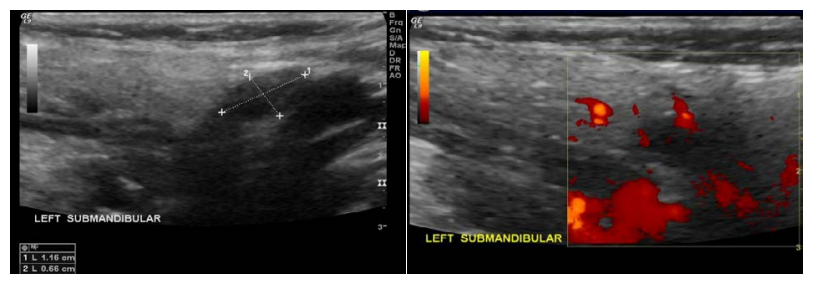

(B): This repeat US, guided by MIBI results, confirmed an enlarged parathyroid gland within the left submandibular gland as the initial US scan did not demonstrate the presence of any enlarged parathyroid glands.

Figure 7. A 63-year-old female presented with a PTH level of $90.2 \mathrm{pg} / \mathrm{ml}$ and a calcium level of $10.2 \mathrm{mg} / \mathrm{dl}$. 


\section{Conclusion}

MIBI provides guidance for the interpretation of US data, especially in the context of ectopic parathyroid adenomas. US offers detailed anatomic information and supports the diagnostic confidence of MIBI in localizing ectopic parathyroid glands, small abnormal parathyroid glands, or concurrent thyroid nodules. US can be particularly helpful in patients with more than one enlarged parathyroid gland. In the majority of cases, the dual utilization of MIBI and US was able to successfully overcome the inherent limitations of each modality.

\section{Authorship and contributorship}

Dr. Yang performed the main study design, and Drs. Yang, Alexander, and Chadha conducted the data collection and analyses. Dr. Yang prepared the manuscript drafts with significant intellectual contribution and critical revisions from Anna Li. All authors have approved the final manuscript.

\section{Acknowledgments}

We would like to express our sincere gratitude to Simon Long, MD for his contributions to the literature review and the nuclear medicine technologists and ultrasound technologists in our Department of Radiology for their support in the data collection for this project.

\section{References}

1. Johnson NA, Tublin ME, Ogilvie JB (2007) Parathyroid imaging: technique and role in the preoperative evaluation of primary hyperparathyroidism. AJR AM J Roentgenol 188: 1706-1715. [Crossref]

2. Kunstman JW, Kirsch JD, Mahajan A, Udelsman R (2013) Parathyroid localization and implications for clinical management. J Clin Endocrinol Metab 98: 902-912. [Crossref]

3. Piciucchi S, Barone D, Gavelli G, Dubini A, Oboldi D, et al. (2012) Primary hyperparathyroidism: imaging to pathology. J Clin Imaging Sci 2: 59. [Crossref]

Copyright: $\odot 2017$ Yang Z. This is an open-access article distributed under the terms of the Creative Commons Attribution License, which permits unrestricted use, distribution, and reproduction in any medium, provided the original author and source are credited. 